\title{
Error Analysis for Absorbing Boundary Conditions
}

\author{
Laurence Halpern ${ }^{1}$ and Jeffrey Rauch ${ }^{2, \star}$ \\ Centre de Mathématiques Appliquées, Ecole Polytechnique, F-91128 Palaiseau, France \\ Department of Mathematics, University of Michigan, Ann Arbor, MI 48109, USA
}

\begin{abstract}
Summary. An estimate is derived for the error committed by the introduction of artificial boundaries and corresponding artificial boundary conditions when solving wave equations on unbounded domains. The estimate has two terms. One is proportional to the largest reflection coefficient for the artificial boundary condition, the maximum taken only on those rays which appear in the computation. The second term is proportional to $1 / k$ where $k$ is a measure of the average frequency present in the solution.
\end{abstract}

Subject Classifications: AMS(MOS): 35L20; CR: G1.8.

\section{$\S 1$. Setting the Problem}

To compute a solution of the wave equation

$$
\mathbf{L} u \equiv \partial^{2} u / \partial t^{2}-\Sigma \partial^{2} u / \partial x_{i}^{2}=0
$$

with $x \in \mathbb{R}^{d}$, one is forced by the finiteness of any computing machine to replace $\mathbb{R}^{d}$ by a bounded subset, $\Omega$. Then, in addition to the Cauchy data, one must impose conditions at the boundary of this compact domain in order to define a unique approximant. The usual idea is the following. In the exterior of $Q$, $u$ satisfies $L u=0$ with vanishing initial data. Let $N$ be the Dirichlet to Neumann operator, $\left.\left.u\right|_{\mathbb{R} \times \partial \Omega} \rightarrow \partial_{v} u\right|_{\mathbb{R} \times \partial \Omega}$. Ideally, one would impose the transparent condition, $\partial_{v} v+N v=0$, at the boundary. Then the solution $v$ would be the exact solution and waves would pass freely through $\partial \Omega$. However, $N$ is nonlocal and this nonlocality, especially in time, makes a time stepping scheme impossible. The boundary conditions chosen in practice are designed to approximate this exact condition. Waves defined by the mixed problem are partially reflected, the boundary is not transparent. The goal is to impose artificial boundary conditions which are weakly reflecting in some sense. Such conditions are often called

* Research partially supported by the U.S. National Science Foundation under grant number DMS8601783 
absorbing since the majority of a wave is absorbed by the boundary and only a small part is reflected. To summarize, a good artificial condition must define a well-posed mixed initial boundary value problem and should be absorbing.

In two important papers, Engquist and Majda [2,3] constructed a family of such boundary conditions whose reflection coefficients (to be defined later) could be made as small as one likes. They were generated by Padé approximants to the exact, or transparent, condition. Taylor polynomial approximations of order higher than one yield ill-posed mixed problems (see also [10]).

It is the goal of this paper to analyze the error committed when using artificial boundary conditions. The error estimate is a sum of two terms. One is proportional to the largest relevant reflection coefficient, and the other is proportional to $1 / k$ where $k$ is a measure of the average frequency in the wave considered. The latter is present because the ideas of geometric optics, in particular the reflection coefficient, are appropriate at high frequency only.

The result, and the proof, are quantitative versions of the now standard qualitative results describing the reflection of singularities.

\section{§ 2. The Artificial Boundary Conditions}

We suppose that Cauchy data are given in a set $\omega \subset \mathbb{R}^{d}$. The computation is then to be performed in the artificial domain $\Omega, \omega \subset \Omega \subset \mathbb{R}^{d}$. Two common choices for $\Omega$ are balls and rectangles. We suppose that $\Omega$ is convex with smooth boundary of strictly positive curvature (unhappily, rectangles do not satisfy this hypothesis). The approximate solution, $v$, is defined in $[0, T] \times \Omega$ as the solution of a mixed initial boundary value problem

$$
\begin{gathered}
\mathbf{L} v=0, \quad \text { in }] 0, T[\times \Omega \\
\mathbf{B} v=0, \quad \text { in }] 0, T[\times \partial \Omega \\
v=u \quad \text { and } \quad v_{t}=u_{t} \text { at }\{t=0\} \times \Omega .
\end{gathered}
$$

Here, $\mathbf{B}$ is a boundary condition given by an operator of order $m$ at $\mathbb{R} \times \partial \Omega$ possibly pseudodifferential in the $\partial \Omega$ variables. Since $\mathbb{R} \times \partial \Omega$ is noncharacteristic for $\mathbf{L}$, we can express any derivative of $v$ in terms of tangential derivatives of $v$ and its outward normal derivative $\partial_{v} v$. Thus, the boundary operator can be written in the form

$$
\mathbf{B}=\mathbf{P} \partial_{v}+\mathbf{Q},
$$

where $\mathbf{P}$ (respectively $\mathbf{Q}$ ) is a pseudodifferential operator of degree $m-1$ (respectively $m$ ) on the boundary $\mathbb{R} \times \partial \Omega$. The operators $\mathbf{P}$ and $\mathbf{Q}$ are assumed to be differential in time, so their symbols are polynomials in $\tau$. The principal symbols of $\mathbf{P}$ and $\mathbf{Q}$ are denoted $P_{m-1}$ and $Q_{m}$.

We suppose that the mixed problem $\mathbf{L}, \mathbf{B}$ is well posed in the sense that for Cauchy data, $v(0, \cdot), v_{t}(0, \cdot) \in C_{0}^{\infty}(\Omega)$ there is a unique solution $v \in C_{(0)}^{\infty}([0, T]$ 
$\times \bar{\Omega})$ and there is a constant, $c$, independent of $v$ so that

$$
\max _{0<t<T} \int_{\Omega}\left|v_{t}(t)\right|^{2}+\left|\nabla_{x} v(t)\right|^{2} d x \leqq c \int_{\Omega}\left|v_{t}(0)\right|^{2}+\left|\nabla_{x} v(0)\right|^{2} d x .
$$

The conditions constructed by Engquist and Majda are well-posed in the even stronger sense of Kreiss [2, 3, 5, 10$]$.

\section{§ 3. The Sets $\Gamma$ and $\Gamma_{b}$ of Important Codirections}

For fixed $T>0$, we want to estimate the difference $u-v$ on $[0, T] \times \bar{\Omega}$. The method is microlocal. Only a subset of the frequencies in $T^{*}([0, T] \times \bar{\Omega})$ are relevant. First notice that the wavefront set $W F u$ is contained in the union of null bicharacteristics of $\mathbf{L}$ which pass over $\omega$. The projections of these null bicharacteristics onto $t, x$ space cut $\mathbb{R} \times \partial \Omega$ transversally thanks to the fact that $\Omega$ is strictly convex. At $q$ in the boundary these bicharacteristics project to points $\tau, \xi^{\prime} \in T_{q}^{*}([0, T] \times \partial \Omega)$ with $\tau^{2}>\left|\xi^{\prime}\right|^{2}$, that is, they lie in the hyperbolic region for $\mathbf{L}$. Over such points pass two bicharacteristics, one leaving $\Omega$ and the second reflected.

The reflected curves will not lie in the wavefront set of $u$, but will usually be carriers of singularities of $v$. These rays may again encounter the boundary where they will be reflected, passing again over hyperbolic points.

Let $\Gamma \subset T^{*}(\mathbb{R} \times \Omega)$ be the set generated by the null bicharacteristics passing over $\omega$ and their reflections. Let $\Gamma_{b}$ be the projection on $T^{*}(\mathbb{R} \times \partial \Omega)$ of the points in closure of $\Gamma$.

We use the standard extension, $W F_{b}$, of the wavefront set, easily defined for solutions of $\mathbf{L} v \in C^{\infty}$ up to the boundary. This subset of $T^{*}(\mathbb{R} \times \Omega) \cup$ $T^{*}(\mathbb{R} \times \partial \Omega)$ is defined to be the ordinary wavefront set over the interior. At the boundary one introduces local coordinates so that $\mathbb{R} \times \Omega=\left\{y_{1}>0\right\}$, $y=\left(y_{1}, y^{\prime}\right)$, and $\eta=\left(\eta_{1}, \eta^{\prime}\right)$ is dual to $y$. Then $\left(y^{\prime}, \eta^{\prime}\right) \notin W F_{b} v$ if and only if there is a pseudodifferential operator $A\left(y^{\prime}, D^{\prime}\right) \in O P S^{0}$, that is the symbol $\sigma(A) \in S^{0}$, properly supported and elliptic at $y^{\prime}, \eta^{\prime}$ such that $A v$ is smooth near $y^{\prime}$. Membership in $H^{s}$ microlocally at $y^{\prime}, \eta^{\prime}$ is defined similarly, $A v \in H^{s}$ near $y^{\prime}$ being the criterion. Now standard results on the transverse reflection of singularities yields

$$
W F_{b} u \cup W F_{b} v \subset \Gamma \cup \Gamma_{b} .
$$

For a computation in $[0, T]$ only the parts of $\Gamma$ and $\Gamma_{b}$ in this time band play a role.

\section{§4. The Reflection Coefficient $r\left(t, x ; \tau, \xi^{\prime}\right)$}

There are many equivalent ways to define the reflection coefficient $r$. We recall two in this section and a third will appear in the proof of the main estimate. The function $r$ is defined on the hyperbolic region, $\left\{\tau^{2}>\left|\xi^{\prime}\right|^{2}\right\} \subset T^{*}(\mathbb{R} \times \partial \Omega)$. 


\section{Plane Wave Definition}

This is the usual treatment in physics texts. For $q \in \mathbb{R} \times \partial \Omega$, choose Euclidean coordinates so that the outward normal to $\partial \Omega$ at $q$ is $(1,0, \ldots, 0)$. Define a homogeneous constant coefficient boundary operator $\mathscr{B}$ by taking the principal symbols $P_{m-1}$ and $Q_{m}$ and freezing the values at $q$.

For $\tau, \xi^{\prime}$ in the hyperbolic region over $q$, the two points in $\operatorname{Char} \mathbf{L} \cap T_{q}^{*}$ which project to $\left(q ; \tau, \xi^{\prime}\right)$ are $\left(q ; \tau, \pm \xi_{1}, \xi^{\prime}\right)$ where $\xi_{1} \equiv\left(1-\left|\xi^{\prime}\right|^{2} / \tau^{2}\right)^{1 / 2} \tau$. Let $w_{ \pm}$ $\equiv e^{i \phi} \pm, \phi_{ \pm} \equiv\left\langle\left(\tau, \pm \xi_{1}, \xi^{\prime}\right),\left(t, x_{1}, x^{\prime}\right)\right\rangle$, be the corresponding exponential solutions of the wave equation. Note that $\tau$ and $\xi_{1}$ have the same sign. Then, $w_{-}$is called the outgoing wave and $w_{+}$incoming, and $r\left(q ; \tau, \xi^{\prime}\right)$ is the unique value such that $w_{-}+r w_{+} \equiv w$ satisfies the boundary condition $\mathscr{B} w=0$.

Combining the definitions of $\mathscr{B}$ and $w$ above yields an equation for $r$,

$$
P_{m-1}\left(q ; \tau, \xi^{\prime}\right) i \xi_{1}(r-1)+Q_{m}\left(q ; \tau, \xi^{\prime}\right)(r+1)=0 .
$$

The coefficient of $r$ is equal to $\mathscr{B} w_{+}$and does not vanish. If it did, then $w_{+}$ would be an incoming plane wave solution of $\mathbf{L} w_{+}=0, \mathscr{B} w_{+}=0$. Starting with such a solution it is not difficult to show that the mixed problem $\mathbf{L}, \mathbf{B}$ is not well-posed in the sense required in $\S 2$.

\section{Geometric Optics Definition}

This treatment places the previous one in an analytically convenient form. Given a point $q \in \mathbb{R} \times \partial \Omega$ and a smooth $\phi$ defined on a neighborhood of $q$ in $\mathbb{R} \times \partial \Omega$ with $\left(\phi_{t}\right)^{2}>\left|\nabla_{x} \phi\right|^{2}$, define two phase functions, $\psi_{ \pm}$on an $\mathbb{R}^{d+1}$ neighborhood of $q$ by

$$
\begin{array}{cc}
\left(\partial_{t} \psi_{ \pm}\right)^{2}=\left|\nabla_{x} \psi_{ \pm}\right|^{2},\left.\quad \psi_{ \pm}\right|_{\mathbb{R} \times \partial \Omega}=\phi, \\
\partial_{v} \psi_{+}=-\partial_{v} \psi_{-} \quad \text { in } R \times \partial \Omega .
\end{array}
$$

The phase $\psi_{-}$corresponding to outgoing waves is the one for which $\partial_{v} \psi$ and $\partial_{t} \psi$ have opposite signs. Given an outgoing asymptotic solution from geometric optics,

$$
w_{-}=a_{-}(t, x, \lambda) e^{i \lambda \psi_{-}}, \quad a_{-} \approx \sum_{n=0}^{\infty} a_{-, n}(t, x) \lambda^{-n}, \quad \mathbf{L} w_{-} \approx 0 .
$$

There is a unique reflected wave,

$$
w_{+}=a_{+}(t, x, \lambda) e^{i \lambda \psi+}, \quad a_{+} \approx \sum_{n=0}^{\infty} a_{+, n}(t, x) \lambda^{-n}, \quad \mathbf{L} w_{+} \approx 0,
$$

so that on $\mathbb{R} \times \partial \Omega, \mathbf{B}\left(w_{+}+w_{-}\right) \approx 0$. On the boundary, the ratio of principal symbols $a_{+, 0}(t, x) / a_{-, 0}(t, x)$ is equal to the reflection coefficient $r(t, x, d \phi)$.

For artificial boundary conditions, the reflected wave represents error. The ratio of reflected to incident wave is measured by $r$. Thus,

$$
\varepsilon \equiv \max \left\{\left|r\left(t, x^{\prime} ; \tau, \xi^{\prime}\right)\right|:\left(t, x ; \tau, \xi^{\prime}\right) \in \Gamma_{b} \text { and } 0 \leqq t \leqq T\right\}
$$


yields an intuitive lower bound on the attainable accuracy. For well designed schemes, $\varepsilon$ should be small.

\section{§. Statement of the Main Theorem}

For solutions of the wave equation on $\mathbb{R}^{d}$, there is a natural family of conserved energies:

$$
e_{s+1} \equiv \int\left|\partial_{t}\left(1-\Delta_{x}\right)^{s / 2} u\right|^{2}+\left|\nabla_{x}\left(1-A_{x}\right)^{s / 2} u\right|^{2} d x .
$$

So long as $u$ is supported in a fixed compact subset of $\mathbb{R}^{d}, e_{s}$ is equivalent to the square of the $H^{s}$ norm. We now state the main error estimate.

Theorem. There is a constant $c_{1}$ depending only on $\omega, \Omega, T, s \in \mathbb{Z}_{+}$and a second constant $c_{2}$ depending also on the absorbing boundary condition $\mathbf{B}$ so that

$$
\|u-v\|_{H^{s}([0, T] \times \Omega)} \leqq c_{1} \varepsilon e_{s}(u)^{1 / 2}+c_{2} e_{s-1}(u)^{1 / 2} .
$$

Remarks. 1. It is crucial that $c_{1}$ is independent of $\mathbf{B}$. Otherwise, the estimate (5.1) would merely affirm the fact that the mixed problem $\mathbf{L}, \mathbf{B}$ is well-posed.

2. Let $k_{s} \geqq 0$ be defined by

$$
k_{s}^{2} \equiv e_{s} / e_{s-1} .
$$

Then, $k_{s}$ is a measure of the average frequency present in the solution $u$, and,

$$
\|u-v\|_{H^{s}}^{2} \leqq 2\left(c_{1} \varepsilon^{2}+c_{2} / k_{s}^{2}\right) e_{s} .
$$

The frequency $k_{s}$ is large for rapidly oscillating solutions or for solutions which have rapid transitions. These are the most important problems for the wave equation. For them, the second term in (5.2) is small so the reflection coefficient is a reasonable measure to use when comparing artificial boundary conditions.

3. The reflection coefficient is unaffected by lower order terms in B. Thus, we provide no guidance in choosing such terms. The ideas of Engquist and Majda yield operators which are homogeneous when the boundary is flat. For curved boundaries, they have a prescription for the lower order terms.

Open Problem. Evaluate the effects of the lower order terms in B when the artificial boundaries are curved.

4. The result and proof extend immediately to variable coefficient operators in place of $\mathbf{L}$. The crucial hypothesis is that null bicharacteristics passing over $\omega$ cross $\mathbb{R} \times \partial \Omega$ over the hyperbolic region. Here, that is guaranteed by the convexity assumption on $\Omega$.

Examples. We first consider the family of boundary operators $\partial_{t}+a \partial_{v}, a>0$. These are all energy nonincreasing. The case $a=0$ is the Dirichlet condition, while the limit $a \rightarrow+\infty$ yields the Neumann condition. The value $a=1$ is the most classical of the absorbing conditions. It is exact in one space dimension. We treat the planar case $x \in \mathbb{R}^{2}$.

To compute the reflection coefficient at angle of incidence $a \in[0, \pi / 2[$ we 
use the plane wave definition. Let $\xi=(\cos \alpha, \sin \alpha), \xi \equiv(-\cos \alpha, \sin \alpha)$. Then, the reflection coefficient is the value $r$ such that

$$
w \equiv e^{i(t-\xi \cdot x)}+r e^{i(t-\xi \cdot x)}
$$

satisfies the boundary condition $\left(\partial_{t}+a \partial / \partial x_{1}\right) w=0$ at $x_{1}=0$. One finds

$$
r=\frac{a \cos \alpha-1}{a \cos \alpha+1}
$$

For the Neumann condition $r \equiv 1$, for Dirichlet $r \equiv-1$. For any of the conditions one has $|r| \leqq 1$ and $r \rightarrow-1$ as $\alpha \rightarrow \pi / 2$. In addition, $|r|$ is a monotonically increasing function of $\alpha$ in $[0, \pi / 2[$ and decreasing in a for $a \in[0,1]$.

If the data are given on $\omega \equiv\left\{|x| \leqq R_{1}\right\}$ and the artificial domain is $\Omega \equiv\{|x|$ $\left.\leqq R_{2}\right\}$ with $R_{1}<R_{2}$, then the maximal angle of incidence occurs for the rays tangent to the smaller disc. They have $\sin \alpha=R_{1} / R_{2}$, so the maximal reflection coefficient is given by,

$$
\varepsilon=\frac{a\left(1-R_{1}^{2} / R_{2}^{2}\right)^{1 / 2}-1}{a\left(1-R_{1}^{2} / R_{2}^{2}\right)^{1 / 2}+1} .
$$

For flat boundaries, the second boundary condition of Engquist and Majda is

$$
\mathbf{B}_{2} u \equiv \frac{\partial}{\partial t} \partial_{v} u+\frac{\partial^{2} u}{\partial s^{2}}-\frac{\partial^{2} u}{\partial t^{2}}=0
$$

where $s$ is arclength in the boundary. For curved boundaries the condition becomes

$$
\frac{\partial}{\partial t} \mathbf{B}_{2} u+\text { lower order terms }=0 \text {. }
$$

The corresponding reflection coefficient has magnitude equal to the square of the coefficient for $\partial_{v}+\partial_{t}[2$, p. 633]. The table below gives values of $\varepsilon$ for two absorbing conditions in the case of concentric discs described above.

$\begin{array}{llll}R_{2} / R_{1} & 2 & 3 & 4 \\ \partial_{t}+\partial_{v} & 0.072 & 0.029 & 0.016 \\ 2^{\text {nd }} E-M \text { cond. } & 0.005 & 0.001 & 0.0002\end{array}$

Values of $\varepsilon$ For Concentric Discs of Radii $R_{1}<R_{2}$.

Computations verify the net advantage of the second condition [2].

\section{§6. Proof of the Theorem}

Let $\delta \equiv \operatorname{dist}(\omega, \partial \Omega)$, then $u=v$ on $[0, \delta[\times \Omega$.

For $u, v$ in $H^{s}([0, T] \times \Omega)$, we estimate the $H^{s}$ norm of the difference $u-v$. Any part dominated by const $\cdot e_{s-1}^{1 / 2}$ can be neglected. Thus, we may suppose that for $0<t<\delta, W F u$ and $W F v$ are supported in a small conic neighborhood 
of the null bicharacteristics passing over $\omega$. A pseudodifferential partition of unity replaces the resulting solution by a finite number of solutions, each supported away from $\mathbb{R} \times \partial \Omega$ for $t<\delta$, and, with wavefront set supported in a small conic neighborhood of a single null bicharacteristic $\gamma_{+}$. We abuse notation by calling the continuations of these solutions $u$ and $v$. They satisfy $\mathbf{L} u \in C^{\infty}$, $\mathbf{L} v \in C^{\infty}$. For $u$ this is on $\mathbb{R}^{d+1}$ and for $v$ on $\mathbb{R} \times \Omega$ supplemented by the homogeneous boundary condition $\mathbf{B} v=0$. The $C^{\infty}$ term is estimated in terms of $e_{s-1}$.

Modulo a lower order, hence negligible, error, $u=v$ until the bicharacteristic $\gamma_{+}$approaches the boundary $\mathbb{R} \times \partial \Omega$. At this boundary the singularity of $u$ passes right through. The singularity of $v$ is reflected. Known results on reflection of singularities proved in increasing generality by Chazarain [1], Lax-Nirenberg [7], Majda-Osher [6], and Taylor [8], show that the wavefront set of the reflected wave is contained in the reflected bicharacteristic. We need to make that result quantitative.

Near the point of reflection $q \in \mathbb{R} \times \partial \Omega$, we introduce local coordinates $y=\left(y_{1}, y^{\prime}\right)$ so that $\mathbb{R} \times \Omega$ becomes $\left\{y_{1}>0\right\}, \partial_{v}$ becomes $-\partial / \partial y_{1}$, and,

$$
\mathbf{L}\left(y, D_{y}\right)=\left(D_{1}\right)^{2}+M\left(y, D^{\prime}\right)+\text { lower order. }
$$

The bicharacteristic $\gamma+$ passes over a point $\left(q ; \eta^{\prime}\right) \in T_{q}^{*}\left(\mathbb{R}^{d}\right)$.

As in [9, Chap. IX] and $[4, \S 24.2]$, we make systematic use of pseudodifferential operators in $y^{\prime}, D^{\prime}$ depending smoothly on $y_{1}$. We use the factorization $(24.2 .5)$ of [4],

$$
\mathbf{L}=\left(D_{1}-A_{+}\left(y, D^{\prime}\right)\right)\left(D_{1}-A_{-}\left(y, D^{\prime}\right)\right)+\beta\left(y, D^{\prime}\right)
$$

with $\beta \in S^{-\infty}$ on a conic neighborhood on $\left(0, q ; \xi^{\prime}\right)$. This factorization, introduced for this purpose by Lax and Nirenberg [7], is used to control the incident waves

which satisfy,

$$
u_{-} \equiv\left(D_{1}-\Lambda_{-}\right) u, \quad v_{-} \equiv\left(D_{1}-\Lambda_{-}\right) v,
$$

$$
\left(D_{1}-\Lambda_{+}\left(y, D^{\prime}\right)\right) u_{-} \in C^{\infty}, \quad\left(D_{1}-A_{+}\left(y, D^{\prime}\right)\right) v_{-} \in C^{\infty} .
$$

The first holds in a full neighborhood of $q$, while the second is valid in $y_{1} \geqq 0$. The $C^{\infty}$ terms have $H^{s-1}$ norms estimated by $e_{s-1}^{1 / 2}$, hence makes a negligible contribution to $u_{-}$. These scalar hyperbolic pseudodifferential equations have $\gamma_{+}$as bicharacteristic. In this way, we see that the $H^{s-1}$ norm of $u_{-}-v_{-}$near $q$ is bounded by $c e_{s-1}^{1 / 2}$. The $H^{s-1}$ norms of $u_{-}, v_{-}$give estimates for the $H^{s}$ norms of the incident parts of $u, v$.

The reflected parts,

$$
u_{+} \equiv\left(D_{1}-\tilde{A}_{+}\right) u, \quad v_{+} \equiv\left(D_{1}-\tilde{A}_{+}\right) v,
$$

are defined using $\tilde{A}_{ \pm}$from the factorization (24.2.6) of [4]. We have,

$$
\left(D_{1}-\tilde{\Lambda}_{-}\right) u_{+} \in C^{\infty}, \quad\left(D_{1}-\tilde{\Lambda}_{-}\right) v_{+} \in C^{\infty},
$$


the first on a neighborhood of $q$ and the second in $y_{1} \geqq 0$. Propagating into $y_{1}<0$, we see that near $q,\left\|u_{+}\right\|_{H^{s}} \leqq c e_{s-1}^{1 / 2}$.

It remains to estimate $v_{+}$near $q$. Thanks to Eq. (6.1), it suffices to estimate the $H^{s-1}$ norm of the trace $\left.v_{+}\right|_{y_{1}=0}$ near $q$. As $\widetilde{A}_{+}+\widetilde{A}_{-} \in O P S^{0}\left(\mathbb{R}_{y}^{d+1} \times \mathbb{R}_{\eta}^{d}\right)$, we see that in $y_{1}=0$

$$
D_{1} v=\left(v_{+}+v_{-}\right) / 2 \text { modulo } H^{s-2} .
$$

$\tilde{\Lambda}_{+}-\tilde{\Lambda}_{-}$is elliptic at $\left(q ; \eta^{\prime}\right)$, its principal symbol is equal to $2 L_{2}\left(q, \eta^{\prime}\right)^{1 / 2}$ which is positive since $q, \eta^{\prime}$ is in the hyperbolic region. Choose $E$ a microlocal parametrix, so

$$
v=E\left(y, D^{\prime}\right)\left(v_{+}-v_{-}\right) / 2 \text { modulo } H^{s-1} .
$$

The boundary condition then reads,

$$
\mathbf{P}\left(v_{+}+v_{-}\right)+\mathbf{Q} E\left(v_{+}-v_{-}\right)=0 \text { modulo } H^{s-m-1} .
$$

Microlocally near $\left(q ; \eta^{\prime}\right)$, this is a pseudodifferential equation for $v_{+}$in terms of $v_{-}$. The solution is

where $R \in O P S^{0}$.

$$
v_{+}=R\left(y, D^{\prime}\right) v_{-} \text {modulo } H^{s-2},
$$

The next step is to identify the symbol of $R$ as the reflection coefficient, a thirs interpretation of this important quantity. Let $\phi, \psi_{ \pm}$be as in (4.1), (4.2), and, let $w_{ \pm}$be defined on a neighborhood of $q$ in $\mathbb{R} \times \bar{\Omega}$ by,

$$
\begin{aligned}
\left(D_{1}-\Lambda_{+}\right) w_{-} \in C^{\infty}, \quad & \left(D_{1}-\tilde{A}_{-}\right) w_{+} \in C^{\infty},\left.\quad w_{-}\right|_{y_{1}=0}=e^{i \lambda \phi}, \\
& \mathbf{B}\left(w_{+}+w_{-}\right) \approx 0 .
\end{aligned}
$$

The function $w_{+}+w_{-}$is a geometric optics solution as in the second characterisation of the reflection coefficient. The principal symbol of the incident wave, $w_{-}$, is identically equal to one by the last equality in (6.3). Thus the principal symbol of the reflected part is equal to the reflection coefficient,

$$
\left.w_{+}\right|_{y_{1}=0}=r\left(y^{\prime}, d \phi\right) e^{i \lambda \phi}+O\left(\lambda^{-1}\right)
$$

On the other hand, the asymptotic expansion of a pseudodifferential operator applied to $e^{i \lambda \phi}$ (see $\left[9, \S\right.$ VIII.7]) yields at $y_{1}=0$,

$$
\begin{aligned}
e^{-i \lambda \phi} \mathbf{Q}\left(w_{+}+w_{-}\right) & =Q_{m}\left(y^{\prime}, d \phi\right)\left(a_{+, 0}\left(y^{\prime}\right)+1\right)+O\left(\lambda^{-1}\right), \\
e^{-i \lambda \phi} D_{1} w_{+} & =\sigma\left(\tilde{\Lambda}_{-}\right)\left(y^{\prime}, d \phi\right) a_{+, 0}\left(y^{\prime}\right)+O\left(\lambda^{-1}\right), \\
e^{-i \lambda \phi} D_{1} w_{-} & =\sigma\left(\Lambda_{+}\right)\left(y^{\prime}, d \phi\right)+O\left(\lambda^{-1}\right) .
\end{aligned}
$$

Plugging $\mathbf{P} \partial_{v}\left(w_{+}+w_{-}\right)=-i \mathbf{P} D_{1}\left(w_{+}+w_{-}\right)$and the above identities into the boundary condition (6.4) and comparing with the definition of $R$ gives,

$$
\left.w_{+}\right|_{y_{1}=0}=\sigma(R)\left(y^{\prime}, d \phi\right) e^{i \lambda \phi}+O\left(\lambda^{-1}\right),
$$

and the identification is complete. 
The sharp Gärding inequality and (6.2) then yield near $q$ in $y_{1}=0$,

$$
\left\|v_{+}\right\|_{H^{s-1}} \leqq \varepsilon\left\|v_{-}\right\|_{H^{s-1}}+c\|v\|_{H^{s-1}} .
$$

Near $q$ in the interior, the component $v_{-}$is then controlled using equation (6.1), yielding

$$
\|\|_{H^{s}}^{2} \leqq\left\|v_{+}\right\|_{H^{s-1}}^{2}+\left\|v_{-}\right\|_{H^{s-1}}^{2}+\|v\|_{H^{s-1}}^{2} \leqq c \varepsilon^{2} e_{s}+c e_{s-1}
$$

in such a neighborhood. Then, standard microlocal techniques apply until the next reflection. At that point, another factor $\varepsilon$ will intervene. One continues with at most a finite number of reflections. The end result is the desired estimate, (5.1).

Acknowledgement. This research was begin while the second author was visiting the Centre de Mathématiques Appliquées at the Ecole Polytechnique, and was completed while the first author was a visitor at the University of Michigan. We gratefully acknowledge the hospitality of these institutions.

\section{References}

1. Chazarain, J.: Construction de la parametrix du problème mixte hyperbolique pour l'équation des ondes. C.R. Acad. Sci. Paris 276, 1213-1215 (1973)

2. Engquist, B., Majda, A.: Absorbing boundary conditions for the numerical simulation of waves. Math. Comput. 139, 629-651 (1977)

3. Engquist, B., Majda, A.: Radiation boundary conditions for acoustic and elastic wave calculations. Commun. Pure Appl. Math. XXXII : 313-375 (1979)

4. Hörmander, L.: The Analysis of Linear Partial Differential Operators III. Berlin, Heidelberg, New York: Springer 1985

5. Kreiss, H.O.: Initial boundary value problems for hyperbolic systems. Commun. Pure Appl. Math. XXIII : 277-298 (1970)

6. Majda, A., Osher, S.: Reflection of singularities at the boundary. Commun. Pure Appl. Math. XXVIII :479-499 (1975)

7. Nirenberg, L.: Lectures on Linear Partial Differential Equations. C.B.M.S. Regional Conf. Ser. No. 17. Am. Math. Soc., Providence R.I., 1973

8. Taylor, M.: Reflection of singularities of solutions to hyperbolic initial boundary value problems. Commun. Pure Appl. Math. XXVIII : 457-478 (1975)

9. Taylor, M., Pseudodifferential Operators. Princeton N.J.: Princeton University Press 1981

10. Trefethen, L.N., Halpern, L.: On the well-posedness of one way wave equations and absorbing boundary conditions. Math. Comput. 176, 421-436 (1986) 